\title{
The $\mathrm{SL}(2, \mathbb{C})$-Character Varieties of Torus Knots
}

\author{
Vicente MUÑOZ \\ Instituto de Ciencias Matemáticas CSIC-UAM-UC3M-UCM \\ Consejo Superior de Investigaciones Científicas \\ Serrano 113 bis \\ 28006 Madrid - Spain \\ vicente.munoz@imaff.cfmac.csic.es \\ Facultad de Matemáticas \\ Universidad Complutense de Madrid \\ Plaza Ciencias 3 \\ 28040 Madrid - Spain
}

Received: September 29, 2008

Accepted: January 13, 2009

\begin{abstract}
Let $G$ be the fundamental group of the complement of the torus knot of type $(m, n)$. This has a presentation $G=\left\langle x, y \mid x^{m}=y^{n}\right\rangle$. We find the geometric description of the character variety $X(G)$ of characters of representations of $G$ into $\mathrm{SL}(2, \mathbb{C})$.
\end{abstract}

Key words: Torus knot, characters, representations.

2000 Mathematics Subject Classification: Primary: 14D20. Secondary: 57M25, 57M27.

\section{Introduction}

Since the foundational work of Culler and Shalen [1], the varieties of SL(2, C)-characters have been extensively studied. Given a manifold $M$, the variety of representations of $\pi_{1}(M)$ into $\mathrm{SL}(2, \mathbb{C})$ and the variety of characters of such representations both contain information of the topology of $M$. This is specially interesting for 3-dimensional manifolds, where the fundamental group and the geometrical properties of the manifold are strongly related.

This can be used to study knots $K \subset S^{3}$, by analysing the $\mathrm{SL}(2, \mathbb{C})$-character variety of the fundamental group of the knot complement $S^{3}-K$. In this paper, we study the case of the torus knots $K_{m, n}$ of any type $(m, n)$. The case $(m, n)=(m, 2)$ was analysed in [3] and the general case was recently determined in [2] by a method different from ours.

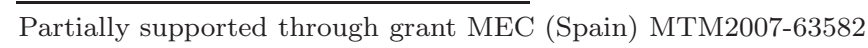




\section{Character varieties}

A representation of a group $G$ in $\operatorname{SL}(2, \mathbb{C})$ is a homomorphism $\rho: G \rightarrow \operatorname{SL}(2, \mathbb{C})$. Consider a finitely presented group $G=\left\langle x_{1}, \ldots, x_{k} \mid r_{1}, \ldots, r_{s}\right\rangle$, and let $\rho: G \rightarrow \operatorname{SL}(2, \mathbb{C})$ be a representation. Then $\rho$ is completely determined by the $k$-tuple $\left(A_{1}, \ldots, A_{k}\right)=$ $\left(\rho\left(x_{1}\right), \ldots, \rho\left(x_{k}\right)\right)$ subject to the relations $r_{j}\left(A_{1}, \ldots, A_{k}\right)=0,1 \leq j \leq s$. Using the natural embedding $\mathrm{SL}(2, \mathbb{C}) \subset \mathbb{C}^{4}$, we can identify the space of representations as

$$
\begin{aligned}
R(G) & =\operatorname{Hom}(G, \operatorname{SL}(2, \mathbb{C})) \\
& =\left\{\left(A_{1}, \ldots, A_{k}\right) \in \operatorname{SL}(2, \mathbb{C})^{k} \mid r_{j}\left(A_{1}, \ldots, A_{k}\right)=0,1 \leq j \leq s\right\} \subset \mathbb{C}^{4 k}
\end{aligned}
$$

Therefore $R(G)$ is an affine algebraic set.

We say that two representations $\rho$ and $\rho^{\prime}$ are equivalent if there exists $P \in \mathrm{SL}(2, \mathbb{C})$ such that $\rho^{\prime}(g)=P^{-1} \rho(g) P$, for every $g \in G$. This produces an action of $\operatorname{SL}(2, \mathbb{C})$ in $R(G)$. The moduli space of representations is the GIT quotient

$$
M(G)=\operatorname{Hom}(G, \operatorname{SL}(2, \mathbb{C})) / / \operatorname{SL}(2, \mathbb{C}) .
$$

A representation $\rho$ is reducible if the elements of $\rho(G)$ all share a common eigenvector, otherwise $\rho$ is irreducible.

Given a representation $\rho: G \rightarrow \operatorname{SL}(2, \mathbb{C})$, we define its character as the map $\chi_{\rho}: G \rightarrow \mathbb{C}, \chi_{\rho}(g)=\operatorname{tr} \rho(g)$. Note that two equivalent representations $\rho$ and $\rho^{\prime}$ have the same character, and the converse is also true if $\rho$ or $\rho^{\prime}$ is irreducible $[1$, Proposition 1.5.2].

There is a character map $\chi: R(G) \rightarrow \mathbb{C}^{G}, \rho \mapsto \chi_{\rho}$, whose image

$$
X(G)=\chi(R(G))
$$

is called the character variety of $G$. Let us give $X(G)$ the structure of an algebraic variety. By the results of [1], there exists a collection $g_{1}, \ldots, g_{a}$ of elements of $G$ such that $\chi_{\rho}$ is determined by $\chi_{\rho}\left(g_{1}\right), \ldots, \chi_{\rho}\left(g_{a}\right)$, for any $\rho$. Such collection gives a map

$$
\Psi: R(G) \rightarrow \mathbb{C}^{a}, \quad \Psi(\rho)=\left(\chi_{\rho}\left(g_{1}\right), \ldots, \chi_{\rho}\left(g_{a}\right)\right) .
$$

We have a bijection $X(G) \cong \Psi(R(G))$. This endows $X(G)$ with the structure of an algebraic variety. Moreover, this is independent of the chosen collection as proved in [1].

Lemma 1.1. The natural algebraic map $M(G) \rightarrow X(G)$ is a bijection.

Proof. The map $R(G) \rightarrow X(G)$ is algebraic and $\mathrm{SL}(2, \mathbb{C})$-invariant, hence it descends to an algebraic map $\varphi: M(G) \rightarrow X(G)$. Let us see that $\varphi$ is a bijection.

For $\rho$ an irreducible representation, if $\varphi(\rho)=\varphi\left(\rho^{\prime}\right)$ then $\rho$ and $\rho^{\prime}$ are equivalent representations; so they represent the same point in $M(G)$.

Now suppose that $\rho$ is reducible. Consider $e_{1} \in \mathbb{C}^{2}$ the common eigenvector of all $\rho(g)$. This gives a sub-representation $\rho^{\prime}: G \rightarrow \mathbb{C}^{*}$ of $G$. We have a quotient 
representation $\rho^{\prime \prime}=\rho / \rho^{\prime}: G \rightarrow \mathbb{C}^{*}$, defined as the representation induced by $\rho$ in the quotient space $\mathbb{C}^{2} /\left\langle e_{1}\right\rangle$. As characters, $\rho^{\prime \prime}=\rho^{\prime-1}$. The representation $\rho^{\prime} \oplus \rho^{\prime \prime}$ is the semisimplification of $\rho$. It is in the closure of the $\operatorname{SL}(2, \mathbb{C})$-orbit through $\rho$. Clearly, $\chi_{\rho}(g)=\rho^{\prime}(g)+\rho^{\prime}(g)^{-1}$. Now if $\rho$ and $\tilde{\rho}$ are two reducible representations and $\varphi(\rho)=\varphi(\tilde{\rho})$, then their semisimplifications have the same character, that is

$$
\chi_{\rho}(g)=\chi_{\tilde{\rho}}(g) \Rightarrow \rho^{\prime}(g)+\rho^{\prime}(g)^{-1}=\tilde{\rho}^{\prime}(g)+\tilde{\rho}^{\prime}(g)^{-1} .
$$

Therefore $\rho^{\prime}=\tilde{\rho}^{\prime}$ or $\rho^{\prime}=\tilde{\rho}^{\prime-1}$. In either case $\rho$ and $\tilde{\rho}$ represent the same point in $M(G)$, which is actually the point represented by $\rho^{\prime} \oplus \rho^{\prime-1}$.

\section{Character varieties of torus knots}

Let $T^{2}=S^{1} \times S^{1}$ be the 2-torus and consider the standard embedding $T^{2} \subset S^{3}$. Let $m, n$ be a pair of coprime positive integers. Identifying $T^{2}$ with the quotient $\mathbb{R}^{2} / \mathbb{Z}^{2}$, the image of the straight line $y=\frac{m}{n} x$ in $T^{2}$ defines the torus knot of type $(m, n)$, which we shall denote as $K_{m, n} \subset S^{3}$ (see [4, Chapter 3]).

For any knot $K \subset S^{3}$, we denote by $G(K)$ the fundamental group of the exterior $S^{3}-K$ of the knot. It is known that

$$
G_{m, n}=G\left(K_{m, n}\right) \cong\left\langle x, y \mid x^{m}=y^{n}\right\rangle .
$$

The purpose of this paper is to describe the character variety $X\left(G_{m, n}\right)$.

In [3], the character variety $X\left(G_{m, 2}\right)$ is computed. We want to extend the result to arbitrary $m, n$, and give a simpler argument than that of [3].

After the completion of this work, we became aware of the paper [2] where the character varieties of $X\left(G_{m, n}\right)$ are determined (even without the assumption of $m, n$ being coprime). However, our method is more direct than the one presented in [2].

To start with, note that

$$
R\left(G_{m, n}\right)=\left\{(A, B) \in \mathrm{SL}(2, \mathbb{C}) \mid A^{m}=B^{n}\right\} .
$$

Therefore we shall identify a representation $\rho$ with a pair of matrices $(A, B)$ satisfying the required relation $A^{m}=B^{n}$.

We decompose the character variety

$$
X\left(G_{m, n}\right)=X_{\text {red }} \cup X_{\text {irr }},
$$

where $X_{\text {red }}$ is the subset consisting of the characters of reducible representations (which is a closed subset by [1]), and $X_{i r r}$ is the closure of the subset consisting of the characters of irreducible representations.

Proposition 2.1. There is an isomorphism $X_{\text {red }} \cong \mathbb{C}$. The correspondence is defined by

$$
\rho=\left(A=\left(\begin{array}{cc}
t^{n} & 0 \\
0 & t^{-n}
\end{array}\right), B=\left(\begin{array}{cc}
t^{m} & 0 \\
0 & t^{-m}
\end{array}\right)\right) \mapsto s=t+t^{-1} \in \mathbb{C} .
$$


Proof. By the discussion in Lemma 1.1, an element in $X_{\text {red }}$ is described as the character of a split representations $\rho=\rho^{\prime} \oplus \rho^{\prime-1}$. This means that in a suitable basis,

$$
A=\left(\begin{array}{cc}
\lambda & 0 \\
0 & \lambda^{-1}
\end{array}\right) \quad \text { and } \quad B=\left(\begin{array}{cc}
\mu & 0 \\
0 & \mu^{-1}
\end{array}\right) .
$$

The equality $A^{m}=B^{n}$ implies $\lambda^{m}=\mu^{n}$. Therefore there is a unique $t \in \mathbb{C}$ with $t \neq 0$ such that

$$
\left\{\begin{array}{l}
\lambda=t^{n} \\
\mu=t^{m}
\end{array}\right.
$$

(Here we use the coprimality of $(m, n))$. Note that the pair $(A, B)$ is well-defined up to permuting the two vectors in the basis. This corresponds to the change $(\lambda, \mu) \mapsto$ $\left(\lambda^{-1}, \mu^{-1}\right)$, which in turn corresponds to $t \mapsto t^{-1}$. So $(A, B)$ is parametrized by $s=t+t^{-1} \in \mathbb{C}$.

Lemma 2.2. Suppose that $\rho=(A, B) \in R\left(G_{m, n}\right)$. In any of the following cases:

(a) $A^{m}=B^{n} \neq \pm \mathrm{Id}$,

(b) $A= \pm \mathrm{Id}$ or $B= \pm \mathrm{Id}$,

(c) $A$ or $B$ is non-diagonalizable,

the representation $\rho$ is reducible.

Proof. First suppose that $A$ is diagonalizable with eigenvalues $\lambda, \lambda^{-1}$, and suppose that $\lambda^{m} \neq \pm 1$. Then there is a basis $e_{1}, e_{2}$ in which $A=\left(\begin{array}{cc}\lambda & 0 \\ 0 & \lambda^{-1}\end{array}\right)$, which is well-determined up to multiplication of the basis vectors by non-zero scalars. Then

$$
B^{n}=A^{m}=\left(\begin{array}{cc}
\lambda^{m} & 0 \\
0 & \lambda^{-m}
\end{array}\right)
$$

is a diagonal matrix, different from $\pm \mathrm{Id}$. Therefore $B$ must be diagonal in the same basis, $B=\left(\begin{array}{cc}\mu & 0 \\ 0 & \mu^{-1}\end{array}\right)$, with $\lambda^{m}=\mu^{n}$. This proves the reducibility in case (a).

Now suppose that $A=\lambda \mathrm{Id}, \lambda= \pm 1$. Then $B^{n}=\lambda^{m} \mathrm{Id}$, so it must be that $B$ is diagonalizable. Using a basis in which $B$ is diagonal, we get the reducibility in case (b).

Finally, suppose that $A$ is not diagonalizable. Then there is a suitable basis on which $A$ takes the form $A=\left(\begin{array}{cc}\lambda & 1 \\ 0 & \lambda\end{array}\right)$, with $\lambda= \pm 1$. Clearly

$$
B^{n}=A^{m}=\lambda^{m}\left(\begin{array}{cc}
1 & m \lambda \\
0 & 1
\end{array}\right)
$$


and so

$$
B=\left(\begin{array}{cc}
\mu & x \\
0 & \mu
\end{array}\right),
$$

with $\mu= \pm 1, \mu^{n}=\lambda^{m}$ and $\mu n x=\lambda m$. In this basis, the vector $e_{1}$ is an eigenvector for both $A$ and $B$. Hence the representation $(A, B)$ is reducible, completing the case (c).

Proposition 2.3. Let $X_{i r r}^{o}$ be the set of irreducible characters, and $X_{\text {irr }}$ its closure. Then

$$
\begin{aligned}
& X_{i r r}^{o} \cong\left\{(\lambda, \mu, r) \mid \lambda^{m}=\mu^{n}= \pm 1, \lambda \neq \pm 1, \mu \neq \pm 1, r \in \mathbb{C}-\{0,1\}\right\} / \mathbb{Z}_{2} \times \mathbb{Z}_{2}, \\
& X_{\text {irr }} \cong\left\{(\lambda, \mu, r) \mid \lambda^{m}=\mu^{n}= \pm 1, \lambda \neq \pm 1, \mu \neq \pm 1, r \in \mathbb{C}\right\} / \mathbb{Z}_{2} \times \mathbb{Z}_{2} .
\end{aligned}
$$

where $\mathbb{Z}_{2} \times \mathbb{Z}_{2}$ acts as $(\lambda, \mu, r) \sim\left(\lambda^{-1}, \mu, 1-r\right) \sim\left(\lambda, \mu^{-1}, 1-r\right) \sim\left(\lambda^{-1}, \mu^{-1}, r\right)$.

Proof. Let $\rho=(A, B)$ be an element of $R\left(G_{m, n}\right)$ which is an irreducible representation. By Lemma $2.2, A$ is diagonalizable but not equal to $\pm \mathrm{Id}$, and $A^{m}= \pm \mathrm{Id}$. So the eigenvalues $\lambda, \lambda^{-1}$ of $A$ satisfy $\lambda^{m}= \pm 1$ and $\lambda \neq \pm 1$. Analogously, $B$ is diagonalizable but not equal to $\pm \mathrm{Id}$, with eigenvalues $\mu, \mu^{-1}$, with $\mu^{n}= \pm 1, \mu \neq \pm 1$. Moreover,

$$
\lambda^{m}=\mu^{n} .
$$

We may choose a basis $\left\{e_{1}, e_{2}\right\}$ under which $A$ diagonalizes. This is well-defined up to multiplication of $e_{1}$ and $e_{2}$ by two non-zero scalars. Let $\left\{f_{1}, f_{2}\right\}$ be a basis under which $B$ diagonalizes, which is well-defined up to multiplication of $f_{1}, f_{2}$ by non-zero scalars. Then $\left\{\left[e_{1}\right],\left[e_{2}\right],\left[f_{1}\right],\left[f_{2}\right]\right\}$ are four points of the projective line $\mathbb{P}^{1}=\mathbb{P}\left(\mathbb{C}^{2}\right)$. Note that the pair $(A, B)$ is irreducible if and only if the four points are different.

The only invariant of four points in $\mathbb{P}^{1}$ is the double ratio

$$
r=\left(\left[e_{1}\right]:\left[e_{2}\right]:\left[f_{1}\right]:\left[f_{2}\right]\right) \in \mathbb{P}^{1}-\{0,1, \infty\}=\mathbb{C}-\{0,1\} .
$$

So $(A, B)$ is parametrized, up to the action of $\operatorname{SL}(2, \mathbb{C})$, by $(\lambda, \mu, r)$. Permuting the two basis vectors $e_{1}, e_{2}$ corresponds to $(\lambda, \mu, r) \mapsto\left(\lambda^{-1}, \mu, 1-r\right)$, since

$$
\left(\left[e_{2}\right]:\left[e_{1}\right]:\left[f_{1}\right]:\left[f_{2}\right]\right)=1-\left(\left[e_{1}\right]:\left[e_{2}\right]:\left[f_{1}\right]:\left[f_{2}\right]\right) .
$$

Analogously, permuting the two basis vectors $f_{1}, f_{2}$ corresponds to

$$
(\lambda, \mu, r) \mapsto\left(\lambda, \mu^{-1}, 1-r\right) .
$$

Note that this gives an action of $\mathbb{Z}_{2} \times \mathbb{Z}_{2}$ and $X_{\text {irr }}^{o}$ is the quotient of the set of $(\lambda, \mu, r)$ as above by this action.

To describe the closure of $X_{i r r}^{o}$, we have to allow $f_{1}$ to coincide with $e_{1}$. This corresponds to $r=1$ (the same happens if $f_{2}$ coincides with $e_{2}$ ). In this case, $e_{1}$ is 
an eigenvector of both $A$ and $B$, so the representation $(A, B)$ has the same character as its semisimplification $\left(A^{\prime}, B^{\prime}\right)$ given by

$$
A^{\prime}=\left(\begin{array}{cc}
\lambda & 0 \\
0 & \lambda^{-1}
\end{array}\right), \quad B^{\prime}=\left(\begin{array}{cc}
\mu & 0 \\
0 & \mu^{-1}
\end{array}\right) .
$$

This means that the point $(\lambda, \mu, 1)$ corresponds under the identification $X_{\text {red }} \cong \mathbb{C}$ given by Proposition 2.1 to $s_{1}=t_{1}+t_{1}^{-1}$, where $t_{1} \in \mathbb{C}$ satisfies

$$
\left\{\begin{array}{l}
\lambda=t_{1}^{n} \\
\mu=t_{1}^{m}
\end{array}\right.
$$

Also, we have to allow $f_{1}$ to coincide with $e_{2}$ (or $f_{2}$ to coincide with $e_{1}$ ). This corresponds to $r=0$. The representation $(A, B)$ has semisimplification $\left(A^{\prime}, B^{\prime}\right)$ where

$$
A^{\prime}=\left(\begin{array}{cc}
\lambda & 0 \\
0 & \lambda^{-1}
\end{array}\right), \quad B^{\prime}=\left(\begin{array}{cc}
\mu^{-1} & 0 \\
0 & \mu
\end{array}\right) .
$$

So the point $(\lambda, \mu, 1)$ corresponds to $s_{0}=t_{0}+t_{0}^{-1} \in X_{\text {red }} \cong \mathbb{C}$, where $t_{0} \in \mathbb{C}$ satisfies

$$
\left\{\begin{array}{l}
\lambda=t_{0}^{n}, \\
\mu^{-1}=t_{0}^{m} .
\end{array}\right.
$$

Proposition 2.3 says that $X_{i r r}$ is a collection of $\frac{(m-1)(n-1)}{2}$ lines. A pair $(\lambda, \mu)$ with $\lambda^{m}= \pm 1$ and $\mu^{n}= \pm 1$ is given as

$$
\lambda=e^{\pi i k / m}, \quad \mu=e^{\pi i k^{\prime} / n},
$$

where $0 \leq k<2 m, 0 \leq k^{\prime}<2 n$. The condition $\lambda \neq \pm 1, \mu \neq \pm 1$ gives $k \neq 0, m$, $k^{\prime} \neq 0, n$. Finally, the $\mathbb{Z}_{2} \times \mathbb{Z}_{2}$-action allows us to restrict to $0<k<m, 0<k^{\prime}<n$. The condition $\lambda^{m}=\mu^{n}$ means that

$$
k \equiv k^{\prime} \quad(\bmod 2) .
$$

Denote by $X_{i r r}^{k, k^{\prime}}$ the line of $X_{i r r}$ corresponding to the values of $k, k^{\prime}$. Then

$$
X_{i r r}=\bigsqcup_{\substack{0<k<m, 0<k^{\prime}<n \\ k \equiv k^{\prime} \\(\bmod 2)}} X_{i r r}^{k, k^{\prime}}
$$

The line $X_{i r r}^{k, k^{\prime}}$ intersects $X_{\text {red }}$ in two points. This gives a collection of $(m-1)(n-1)$ points in $X_{\text {red }}$, which are defined as follows: under the identification $X_{\text {red }} \cong \mathbb{C}$, these are the points $s_{l}=t_{l}+t_{l}^{-1}$, where

$$
t_{l}=e^{\pi i l / n m},
$$




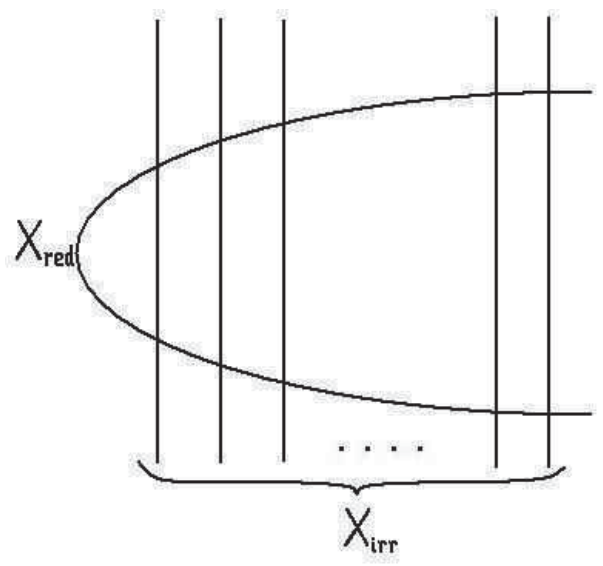

Figure 1 - Picture of $X\left(G_{m, n}\right)$.

and $0<l<m n, m \nmid l, n \nmid l$. Assume that $n$ is odd (note that either $m$ or $n$ should be odd). Then from (1) and (2), the line $X_{i r r}^{k, k^{\prime}}$ intersects at the points $s_{l_{0}}, s_{l_{1}} \in X_{r e d}$ where

$$
\begin{array}{lll}
n l_{0} \equiv k & (\bmod m), & m l_{0} \equiv n-k^{\prime} \quad(\bmod n) \\
n l_{1} \equiv k & (\bmod m), & m l_{1} \equiv k^{\prime} \quad(\bmod n) .
\end{array}
$$

These two points are different since $k^{\prime} \not \equiv n-k^{\prime}(\bmod n)$, as $n$ is odd.

In the case $(m, n)=(2, n)$, this result coincides with [3, Corollary 4.2].

\section{The algebraic structure of $X\left(G_{m, n}\right)$}

We want to give a geometric realization of $X\left(G_{m, n}\right)$ which shows that the algebraic structure of this variety is that of a collection of rational lines as in Figure 1 intersecting with nodal curve singularities.

The map $R\left(G_{m, n}\right) \rightarrow \mathbb{C}^{3}, \rho=(A, B) \mapsto(\operatorname{tr}(A), \operatorname{tr}(B), \operatorname{tr}(A B))$, defines a map

$$
\Psi: X\left(G_{m, n}\right) \rightarrow \mathbb{C}^{3}
$$

Theorem 3.1. The map $\Psi$ is an isomorphism with its image $C=\Psi\left(X\left(G_{m, n}\right)\right)$. $C$ is a curve consisting of $\frac{(n-1)(m-1)}{2}+1$ irreducible components, all of them smooth and isomorphic to $\mathbb{C}$. They intersect with nodal normal crossing singularities following the pattern in Figure 1. 
Proof. Let us look first at $\Psi_{0}=\left.\Psi\right|_{X_{\text {red }}}: X_{\text {red }} \rightarrow \mathbb{C}^{3}$. For a given $\rho=(A, B) \in X_{\text {red }}$, with the shape given in Proposition 2.1, we have that

$$
\Psi_{0}: s=t+t^{-1} \mapsto\left(t^{n}+t^{-n}, t^{m}+t^{-m}, t^{n+m}+t^{-(n+m)}\right) .
$$

This map is clearly injective: the image recovers

$$
\left\{t^{n}, t^{-n}\right\},\left\{t^{m}, t^{-m}\right\},\left\{t^{n+m}, t^{-(n+m)}\right\} .
$$

From this, we recover $\left\{\left(t^{n}, t^{m}\right),\left(t^{-n}, t^{-m}\right)\right\}$ and hence the pair $t, t^{-1}$ (since $n, m$ are coprime).

Let us see that $\Psi_{0}$ is an immersion. The differential is

$$
\frac{d \Psi_{0}}{d t}=\left(n t^{-n-1}\left(t^{2 n}-1\right), m t^{-m-1}\left(t^{2 m}-1\right),(n+m) t^{-n-m-1}\left(t^{2 n+2 m}-1\right)\right) .
$$

This is non-zero at all $t \neq \pm 1$. As $\frac{d s}{d t} \neq 0$, we have $\frac{d \Psi_{0}}{d s} \neq(0,0,0)$. For $t= \pm 1$, we note that $\frac{d s}{d t}=t^{-2}\left(t^{2}-1\right)$, so

$$
\frac{d \Psi_{0}}{d s}=\left(n t^{-n+1} \frac{t^{2 n}-1}{t^{2}-1}, m t^{-m+1} \frac{t^{2 m}-1}{t^{2}-1},(n+m) t^{-n-m+1} \frac{t^{2 n+2 m}-1}{t^{2}-1}\right),
$$

which is non-zero again.

Now, consider a component of $X_{i r r}$ corresponding to a pair $(\lambda, \mu)$. Take $r \in \mathbb{C}$. Fix the basis $\left\{e_{1}, e_{2}\right\}$ of $\mathbb{C}^{2}$ which is given as the eigenbasis of $A$. Let $\left\{f_{1}, f_{2}\right\}$ be the eigenbasis of $B$. As the double ratio $(0: \infty: 1: r /(r-1))=r$, we can take $f_{1}=(1,1)$ and $f_{2}=(r-1, r)$. This corresponds to the matrices:

$$
\begin{aligned}
A & =\left(\begin{array}{cc}
\lambda & 0 \\
0 & \lambda^{-1}
\end{array}\right), \\
B & =\left(\begin{array}{cc}
1 & r-1 \\
1 & r
\end{array}\right)\left(\begin{array}{cc}
\mu & 0 \\
0 & \mu^{-1}
\end{array}\right)\left(\begin{array}{cc}
1 & r-1 \\
1 & r
\end{array}\right)^{-1} \\
& =\left(\begin{array}{cc}
r\left(\mu-\mu^{-1}\right)+\mu^{-1} & (1-r)\left(\mu-\mu^{-1}\right) \\
r\left(\mu-\mu^{-1}\right) & \mu-r\left(\mu-\mu^{-1}\right)
\end{array}\right) .
\end{aligned}
$$

Therefore:

$$
\begin{aligned}
\Psi(A, B) & =(\operatorname{tr}(A), \operatorname{tr}(B), \operatorname{tr}(A B)) \\
& =\left(\lambda+\lambda^{-1}, \mu^{-1}+\mu,\left(\lambda \mu^{-1}+\lambda^{-1} \mu\right)+r\left(\lambda-\lambda^{-1}\right)\left(\mu-\mu^{-1}\right)\right) .
\end{aligned}
$$

The image of this component is a line in $\mathbb{C}^{3}$. Its direction vector is $(0,0,1)$. At an intersection point with $\Psi_{0}\left(X_{r e d}\right)$, the tangent vector to $\Psi_{0}\left(X_{r e d}\right)$, given in (3), has non-zero first and second component, since $\lambda=t^{n}, \mu=t^{m}$ and $t \neq 0, \lambda^{2} \neq 1, \mu^{2} \neq 1$. So the intersection of these components is a transverse nodal singularity.

Finally, note that the map $\Psi: X\left(G_{m, n}\right) \rightarrow C$ is an algebraic map, it is a bijection, and $C$ is a nodal curve (the mildest possible type of singularities). Therefore $\Psi$ must be an isomorphism. 
Corollary 3.2. $M(G) \cong X(G)$, for $G=G_{m, n}$.

Proof. By Lemma 1.1, $\varphi: M(G) \rightarrow X(G)$ is an algebraic map which is a bijection. As the singularities of $X(G)$ are just transverse nodes, $\varphi$ must be an isomorphism.

Acknowledgement. The author wishes to thank the referee for useful comments, specially for pointing out the reference [2].

\section{References}

[1] M. Culler and P. B. Shalen, Varieties of group representations and splittings of 3-manifolds, Ann. of Math. (2) 117 (1983), no. 1, 109-146.

[2] J. Martín-Morales and A-M. Oller-Marcén, On the varieties of representations and characters of a family of one-relator subgroups, arXiv:0805.4716.

[3] A-M. Oller-Marcén, The SL $(2, \mathbb{C})$ character variety of a class of torus knots, Extracta Math. 23 (2008), no. 2, 163-172.

[4] D. Rolfsen, Knots and links, Mathematics Lecture Series, vol. 7, Publish or Perish Inc., Houston, TX, 1990. Corrected reprint of the 1976 original. 